\title{
Distributionally Robust Microgrid Formation Approach for Service Restoration under Random Contingency
}

Cai, Sheng; Xie, Yunyun; Wu, Qiuwei; Zhang, Menglin; Jin, Xiaolong; Xiang, Zhengrong

Published in:

IEEE Transactions on Smart Grid

Link to article, DOI:

10.1109/TSG.2021.3095485

Publication date:

2021

Document Version

Publisher's PDF, also known as Version of record

Link back to DTU Orbit

Citation (APA):

Cai, S., Xie, Y., Wu, Q., Zhang, M., Jin, X., \& Xiang, Z. (2021). Distributionally Robust Microgrid Formation Approach for Service Restoration under Random Contingency. IEEE Transactions on Smart Grid, 12(6), 4926 4937. https://doi.org/10.1109/TSG.2021.3095485

\section{General rights}

Copyright and moral rights for the publications made accessible in the public portal are retained by the authors and/or other copyright owners and it is a condition of accessing publications that users recognise and abide by the legal requirements associated with these rights.

- Users may download and print one copy of any publication from the public portal for the purpose of private study or research.

- You may not further distribute the material or use it for any profit-making activity or commercial gain

- You may freely distribute the URL identifying the publication in the public portal 


\title{
Distributionally Robust Microgrid Formation Approach for Service Restoration under Random Contingency
}

\author{
Sheng Cai, Student Member, IEEE, Yunyun Xie, Member, IEEE, Qiuwei Wu, Senior Member, IEEE, \\ Menglin Zhang, Member, IEEE, Xiaolong Jin, Member, IEEE, Zhengrong Xiang, Member, IEEE
}

\begin{abstract}
When a major outage occurs in a distribution system due to extreme events, service restoration (SR) strategies pick up critical loads after isolating the faults that have occurred. However, in extreme conditions, traditional SR strategies could pose potential security risks to restored services due to subsequent contingencies in succeeding events. To address this challenge, we propose a microgrid-based SR methodology that aims to enhance the preparedness of microgrids during unfolding extreme events. The proposed SR strategy comprises microgrid formation (MF) and sequential service restoration (SSR) steps. The MF makes the benefit of topology switching, generator allocation, and load demand response. Additionally, the uncertainty of line failure probability is considered, and a distributionally robust optimization model is proposed to maximize the expected load restoration with regard to the worst-case distribution of contingencies. Then, the SSR is formulated as a mixed-integer linear program model to yield proper load switching sequences and generation of power sources for sequentially restoring the outage system. The proposed SR measure enhances the system resilience by the proactive formation of microgrids, reducing the impact of cascading phenomenon when lines with high failure probability are tripped. The effectiveness of the proposed method is validated by numerical simulations.
\end{abstract}

Index Terms -Distributionally robust optimization, extreme events, microgrids, sequential service restoration.

\section{NOMENCLATURE}

A. Sets and Indices

$M, m \quad$ Set/Index of MGs

$K, k \quad$ Set/Index of DGs

$K_{\mathrm{f}}, K_{\mathrm{m}} \quad$ Set of fixed/mobile DGs

$K_{\mathrm{b}} \quad$ Set of DGs with black-start capacity

$N, L \quad$ Set of nodes and lines

$N_{\mathrm{m}} \quad$ Set of nodes in MG $m$.

$N^{\prime}, L^{\prime} \quad$ Set of damaged nodes and lines

This work was supported by the National Natural Science Foundation (NNSF) of China under Grants 51977111 and 61773214.

S. Cai, Y. Xie and Z. Xiang are with Nanjing University of Science and Technology, Nanjing, 210094 China (e-mail: njustauto_cs@163.com; yunyun_xie@njust.edu.cn; xiangzr@njust.edu.cn).

Q. Wu and M. Zhang are with Centre for Electric Power and Energy, Department of Electrical Engineering, Technical University of Denmark, Kgs. Lyngby, Denmark (email: qw@elektro.dtu.dk; xiajin@elektro.dtu.dk; menzh@elektro.dtu.dk).

X. Jin is with Key Laboratory of Smart Grid of Ministry of Education, Tianjin University, Tianjin 30072, China (e-mail: xljin@tju.edu.cn).

\section{B. Parameters and Constants}

$n^{m} \quad$ Total number of nodes in MG $m$.

$w_{i} \quad$ Weighting factor of load at node $i$

$\Delta t \quad$ Length of time interval

$D_{i}^{p}, D_{i}^{q} \quad$ Active/reactive load demand at node $i$

$V_{0} \quad$ Reference value of node voltage

$R_{i j}, X_{i j} \quad$ Resistance/reactance of line $(i, j)$

$\varepsilon \quad$ Allowable deviation of node voltage

$P_{k \text { min/max }} \quad$ Minimum/maximum active generation of DG $k$

$Q_{k \cdot \min / \max }$ Minimum/maximum reactive generation of DG $k$

$R_{k}^{\text {ramp }} \quad$ Maximum ramp rate for DG $k$

$T_{i j}^{p}, T_{i j}^{q} \quad$ Maximum active/reactive power flow on line $(i, j)$

$L_{i j} \quad$ Connectivity coefficient between nodes $i$ and $j$

$T_{i i}^{R} \quad$ Time for energization agent start to travel from node $i$

$T_{n} \quad$ Maximum restoration time

$T_{h i}^{R} \quad$ Time for energization agent travels from node $h$ to $i$.

$\lambda_{i}^{U} \quad$ Undiversified loading factor for load at node $i$

$\Delta P d_{i}(l) \quad$ Difference of active demand between two samples.

$\Delta Q d_{i}(l) \quad$ Difference of reactive demand between two samples.

$M_{H} \quad$ Inertia of the MG

$c_{g} \quad$ Ramp-rate of DG response

$f^{0}, f^{\min } \quad$ Reference/minimum frequency of the system

C. Variables

$v_{i}^{m}, c_{i j}^{m} \quad$ Partition state of node $i$ / line $(i, j)$

$z_{i}^{k} \quad$ Connection state of DG $k$ to node $i$

$b_{i j}, y_{i j} \quad$ Switch/interruption state of line $(i, j)$

$s_{i}^{p}, s_{i}^{q} \quad$ Active/reactive load shedding at node $i$

$p_{i j}, q_{i j} \quad$ Active/reactive power flow across line $(i, j)$

$P g_{i}, Q g_{i} \quad$ Active/reactive power generation of DG at node $i$

$V_{i} \quad$ Node voltage at node $i$.

$\delta_{i j} \quad$ Slack variable of line $(i, j)$

$P d_{i, t}, Q d_{i, t} \quad$ Active/reactive load restored at node $i$ at time step $t$.

$x_{i j}^{R . k} \quad$ Binary indicating if restoration path start from $i$ to $j$

$t_{i} \quad$ Time when energization agent arrives at node $i$

$u_{i . t}, u_{i j . t} \quad$ Energization state of node $i /$ line $(i, j)$ at time step $t$ 


\section{INTRODUCTION}

$\mathrm{T}$ ODAY'S power grids are facing an increasing frequency and a higher intensity of outages caused by extreme events (e.g., natural disasters or physical attacks). It is estimated that the weather-related outages cost the U.S. between $\$ 20$ and $\$ 55$ billion annually [1]. Such extreme events are classified as high-impact low-probability (HILP) incidents. The tremendous socioeconomic losses due to HILP incidents underscore the need to address their consequences in a proactive manner [2] and rapidly restore the functionality of power systems from degraded situations, which is defined as grid resilience [3].

Resilience-enhancement measures can be categorized into hardening-oriented and operation-oriented measures [4]. The former aims to reinforce the vulnerable components and make it stronger during HILP incidents [5-7]. Operation-oriented measures refer to proactive or passive strategies taken to make the system capable of dealing with the unfavorable condition as it unfolds [8]. Among the available resilience-enhancement measures, microgrid (MG) is considered as one of the viable and effective solutions due to its ability of islanding [9]. In [10], [11], post-disaster service restoration (SR) schemes were studied to restore critical loads in networked MGs. Refs. [12-14] developed MG formation models to divide the on-outage area into multiple self-supplied MGs. However, in the above studies, the distributed generators (DGs) are pre-positioned. Refs. [15], [16] utilized both fixed and mobile emergency resources (MERs) to form MGs, proving that more loads can be restored with more flexible resources. Some other studies formulated the SR as two-stage models [17], where the first stage decided the network topology and the second stage determined the switching sequences toward the final network configuration.

The aforementioned works are under the premise that all the faulted components have been isolated, and that no more faults will occur in succeeding events. Then, the SR scheme is formed to promptly restore the system. However, extreme events can impose subsequent damage to systems and subject the initially formed MGs to additional risk of collapse. Take Hurricane Katrina as an example, the resulting flooding caused subsequent damage to electricity infrastructures in Texas. Also the physical or cyber incidents (e.g., the Venezuela grid attack in 2020) can launch multistage attacks to impose extended damage to the system [18]. To enhance system preparedness in succeeding events, operation-oriented proactive measures should be taken to moderate the system vulnerability at the event onset [19]. When taking into account the contingency in succeeding events, additional problems emerge for traditional SR strategies, both in the modeling and solution approach.

From the modeling perspective, the system security cannot always be guaranteed if system uncertainties are neglected. The traditional work formulates the SR as deterministic models [20]. However, such an approach could lead to the violation of security constraints when the accurate forecast of renewable energy generation and load demands are unavailable. To deal with this problem, such continuous uncertainties are modeled by introducing uncertainty sets to obtain a more robust SR scheme [21]. However, discrete uncertainties, such as random contingency (e.g., outage of transmission lines and generators), also pose a security risk to the system operation. Although extensive studies [6], [22] have been conducted to calculate the component failure probabilities under extreme events, it is still difficult to estimate an accurate probability distribution. To model uncertainties of contingency, [23] formulated a security evaluation subproblem to explore possible violations when considering wind/load variations and $N-k$ contingencies. However, a proper SR scheme cannot be derived with an uncertainty set considering only the $N-k$ security criterion but not contingency probability distributions. For example, even though [23] identified the worst-case contingency scenario, the probability of such a scenario occurring is quite small.

From the solution perspective, different approaches have been used to solve the problem considering random contingencies. Stochastic programming (SP) [7],[24] is an important way to estimate the contingency's likelihood of occurring, and then generate a set of scenarios to represent the stochasticity in decision making. For example, stochastic pre-disturbance microgird formation (MF) models were given in [25] to reduce the expected amount of load shedding with the consideration of subsequent damage. However, exponentially many scenarios are needed to represent random contingencies when considering the $N-k$ security criterion, which significantly increases the computational burden. Another effective approach is to apply the robust optimization (RO) method [26] to find the most critical contingencies that have the largest impacts on the system. In [27], robust models were presented to mitigate systems' vulnerability by coordinating proactive measures and post-outage recovery measures. However, the RO approach focuses on the worst-case scenario and completely neglects the probabilistic characteristics of contingencies, which may yield over-conservative solutions. For the uncertain contingency, an ambiguity set of contingency probability distributions that satisfy the $N-k$ security criterion can be constructed, and the proposed model can be solved by the distributionally robust (DR) method [28-30]. Compared to the RO, the DR focuses on the probability of contingency occurring. It yields a solution under the worst-case probability distributions, which is less conservative than the RO approach.

In this paper, an effective SR strategy is proposed to form MGs and restore critical loads when considering the random contingencies during extreme events. A two-stage framework is proposed to restore the distribution system with an outage, where the MF stage optimally locates MERs and proactively forms MGs, and the SSR stage determines proper switching actions and dispatches power sources to sequentially restore critical loads. Moreover, considering the uncertainty of contingencies, a DR model is formulated and an effective solution algorithm is employed to rapidly solve it. As such, MG-based SR methods are further improved by enhancing the preparedness of MGs to deal with potential contingencies. Compared with the existing work, this paper has three main contributions:

1. A two-stage SR method is proposed for MER allocation, MG formation and SSR in distribution systems. Compared to the existing MG formation methods, potential contingencies are considered for enhancing system resilience during extreme 
events.

2. The DR model is formulated to maximize the expected load restoration, where an ambiguity set of HILP contingency is constructed, and both the line failure probability and the corresponding consequence are considered. In comparison with the RO method, the proposed DR approach can achieve a less conservative solution.

3. The proposed DR model is transformed into an equivalent RO model, which can be effectively solved through the C\&CG algorithm. The proposed solution algorithm can converge to the global optimal solution. Besides, the probability of the worst-case contingencies is also calculated.

The remainder of this paper is organized as follows: In Section II, the framework of the proposed two-stage SR scheme is described. The mathematical formulations for MF and SSR are introduced in Section III. In Section IV, the solution methodology is presented. The proposed method is validated by detailed simulations of the IEEE 37-node system in Section V. Finally, Section VI concludes the paper.

\section{CONCEPTUAL FRAMEWORK}

\section{A. Principle of $M G$-Based SR}

Topology analyses are performed to illustrate the principle of the proposed MG-based SR approach. The distribution system is defined by a graph-theory notation $G\{N, L\}$, where $N$ and $L$ stand for the sets of nodes and lines, respectively. After extreme events, there might be multiple damage left, and the resulting post-event graph is denoted by $G^{\prime}\left\{N^{\prime}, L^{\prime}\right\}$, where $N^{\prime}$ and $L^{\prime}$ stand for the sets of undamaged nodes and lines. Moreover, since extreme events may launch subsequent damage to the system, set $\widehat{L} \subseteq L^{\prime}$ is defined to represent the faulty lines in succeeding events. We assume that the graphs $G$ and $G^{\prime}$ are observable i.e., the sets and subsets $N, N^{\prime}, L, L^{\prime}$ are known, however, the set $\hat{L}$ is uncertain and unknown when developing the SR scheme.

In SR, MGs are formed in the set $M$. Each MG $m \in M$ includes nodes $N_{\mathrm{m}} \subseteq N^{\prime}$ and lines $L_{\mathrm{m}} \subseteq L^{\prime}$. Two MGs $m_{l}$ and $m_{2}$ satisfy $N_{\mathrm{m} 1} \cap N_{\mathrm{m} 2}=\varnothing$ and $L_{\mathrm{m} 1} \cap L_{\mathrm{m} 2}=\varnothing$. We designate the set of DGs by $K$. Given that DGs can be fixed or mobile, the subsets $K_{\mathrm{f}}, K_{\mathrm{m}} \subseteq K$ represent the sets of fixed and mobile DGs respectively, with $K_{\mathrm{f}} \cap K_{\mathrm{m}}=\varnothing$ and $K_{\mathrm{f}} \cup K_{\mathrm{m}}=K$, and $K_{b}$ represents the DGs with black-start capability.

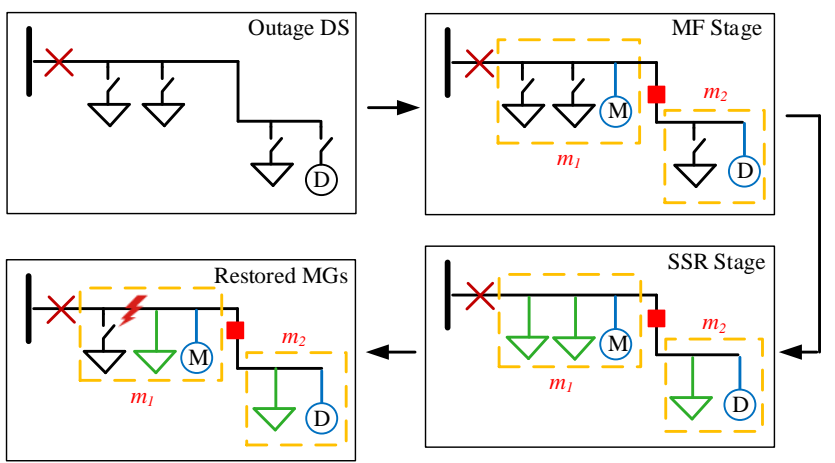

X damaged line $\quad$ subsequent damage $-\smile$ breaker $\square$ line switch

(D) fixed DG (M) mobile DG $\frac{1}{\boldsymbol{\prime}}$ out-of-service load $\downarrow$ restored load

Fig. 1. The MG-based DSR process in a distribution system
Fig.1 illustrates the proposed SR process. Once the distribution system loses the grid supply due to the first extreme event, MER are optimally connected to candidate nodes, and the outage area is sectionalized by opening line switches. After the MG topology is determined, loads are restored sequentially, and multiple parallel restored MGs (i.e. $m_{1}$ and $m_{2}$ ) are finally formed. Moreover, the proposed SR method considers the line interruption in restored MGs during unfolding extreme events, therefore, potential contingency is taken into consideration when determining the optimal MG topology.

\section{B. Two-Stage SR Framework}

In this paper, we propose a two-stage SR scheme, of which the framework is shown in Fig. 2. Firstly, MERs are positioned, and MGs are proactively formed. Secondly, the intermediate switching sequences are generated to restore the system sequentially without violating any operation constraints.

MF Stage: After an outage resulting from extreme events, the parameters (e.g. DS topology, DG capacity and load demand) needed are collected by the outage management system. Moreover, the ambiguity set of contingencies caused by subsequent damage is also predicted through the analysis of historical data. After all the required parameters are obtained, the proposed MF model is solved, and the MG topology is determined by controlling the line switches and locating MERs.

SSR Stage: After the MG topology is determined, parameters including the operating time of switches, DG parameters, load demands, etc. are collected, and the proposed SSR model will be solved. Through the optimization of the switching actions, restoration paths are generated. DGs with black-start capability are deployed to provide cranking power to other power sources without this capability and sequentially pick up critical loads.

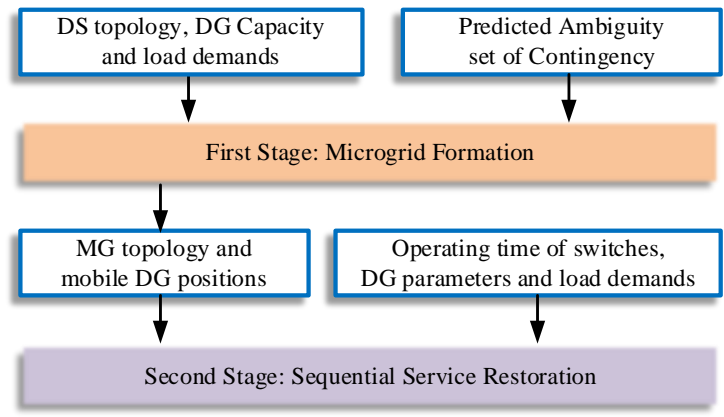

Fig. 2. The proposed two-stage SR framework

\section{Assumptions}

To simplify the SR problem and ensure the feasibility of the solution, several modifications and assumptions have been made as follows:

1) The distribution system is modified to be a balanced system, which has been widely used to study resilience enhancement of distribution systems in extreme conditions [13-17]. The unbalanced condition, which is more practical to implement in real systems, will be considered in further work.

2) We assume that DGs with black-start capacity can expand their coverage to supply loads by separately forming MGs. This assumption is based on the fact that the value of MG with DGs to enhance system resilience has been recognized 
[12],[30]. Under this assumption, the set of MGs id denoted by $\boldsymbol{M}$, and each MG $m \in \boldsymbol{M}$ consists of set of nodes. For reducing the system outage duration, each MG contains only one black- start DG, so we get $|\boldsymbol{M}|=\left|K_{b}\right|$.

3) This paper aims to study the SR scheme to sequentially restore critical loads in a radial distribution system after major faults at the main grid, which occurs frequently under extreme disaster events [12]. Therefore, the distribution system is assumed to be completely de-energized due to the first strike of the natural disaster. Handling the local outage condition will be considered as part of further work.

\section{MATHEMATICAL FORMULATION}

\section{A. DR Optimization Model for MF}

Our DR model aims to find an optimal MG configuration to maximize the load restoration when considering the upcoming disturbances. The two-stage DR model is presented as follows:

1) Objective Function: The highest priority for an outage distribution system is to maximize the total priority weighted load restoration. Considering the subsequent random contingencies, the objective function is formulated as follows:

$$
\max _{g \in G} \min _{\mathbb{P} \in D} E_{\mathbb{P}}[Q(g, y)]
$$

where objective function (1) seeks the worst-case distribution of contingency and the corresponding expected load restoration $Q(g, y)$. And $g:=(v, z, c, b)$ indicates the MG configuration and DG allocation decision variables. $D$ is the ambiguity set of contingencies, which will be elaborated in Section IV. And $Q(g, y)$ represents the worst-case expected load restoration for a given MG topology $g$ and realized contingency $y_{m n}$.

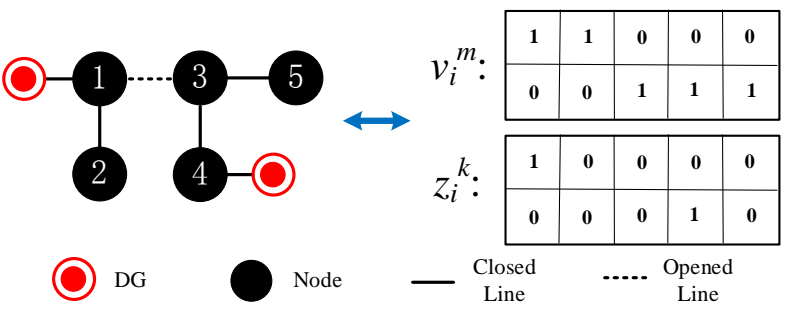

Fig. 3. Example of a MG topology

2) MG Topology Constraints: The main difference between the proposed MG topology constraints and those in existing work lies in the MER position constraints (3)-(6). An example of a MG topology is shown in Fig. 3. $v_{i}$ and $z_{i}$ are the decision variables to be determined. Since there are two black-start DGs, the system is optimally divided into two MGs. The term $v_{i}^{m}=1$ represents node $i$ belong to $m_{t h}$ MG. The term $z_{i}^{k}=1$ indicates that node $i$ is connected by the $k_{t h}$ DG. Compared to the MF model in [16], there is no need to assume a single DG per microgrid. To ensure the radiality of MGs, the following topology constraints should be satisfied:

$$
\begin{gathered}
\sum_{m \in M} v_{i}^{m} \leq 1, \forall i \in N^{\prime} \\
\sum_{i \in N^{\prime}} z_{i}^{k} \leq 1, \forall k \in K \\
\sum_{k \in K_{b}} z_{i}^{k} \leq 1, \forall i \in N^{\prime} \\
v_{i}^{m} \geq z_{i}^{k}, \forall i \in N^{\prime}, k \in K_{b}
\end{gathered}
$$

$$
\begin{gathered}
z_{i}^{k}=1, \forall i \in n^{k}, k \in K_{f} \\
c_{i j}^{m}=v_{i}^{m} \cdot v_{j}^{m}, \forall(i, j) \in L^{\prime}, m \in M \\
b_{i j}=\sum_{m \in M} c_{i j}^{m}, \forall(i, j) \in L^{\prime} \\
\sum_{m \in M} v_{i}^{m}=0, \forall i \in N \backslash N^{\prime} \\
b_{i j}=0, \forall(i, j) \in L \backslash L^{\prime} \\
\sum_{(i, j) \in L \backslash L^{\prime}}\left(1-b_{i j}\right) \leq|M|-1
\end{gathered}
$$

where (2) ensures that each node can only be assigned to one MG. (3) guarantees that each DG can only be connected to one node, and (4) ensures that each node can only contain one DG. (5) indicates that for node $i$ at which the black-start DG $k$ is installed, node $i$ is assigned to the MG formed around the corresponding DG. (6) enforces that the fixed DG is located at node $n^{k}$. (7) indicates that line $(i, j)$ can be assigned to the $m_{t h}$ MG, and only both of its end-nodes $i$ and $j$ belong to this MG. In other words, the line switch $b_{i j}$ is forced to open if nodes $i$ and $j$ are not assigned to the same MG. (8) represents the state of the line switch (i.e., $b_{i j}=0$ represents that the line switch is open, and $b_{i j}=1$ represents that the line switch is closed). (9) and (10) indicate that the faulty nodes and lines cannot be assigned to any MGs during the SR process. (11) indicates that the number of controllable switch lines in 'open' state should be less than $|\boldsymbol{M}|$-1, which can guarantee the radial structure in each MG.

3) Post-Disturbance Rescheduling Process: After the disturbances, a number of corrective actions need to be performed to maximize the load restoration. Such actions include rescheduling the DGs and dispatchable loads. The optimal objective value of the $Q(g, y)$ is formulated as follows:

$$
\begin{gathered}
Q(g, y)=\max _{u \in H(g, y)} \sum_{n \in N^{\prime}} w_{i} \cdot\left(D_{i}^{p}-s_{i}^{p}\right) \\
\text { s.t. } \sum_{j:(j, i) \in L^{\prime}} p_{j i}-\sum_{j:(i, j) \in L^{\prime}} p_{i j}+P g_{i}-D_{i}^{p}+s_{i}^{p}=0, \forall i \in N^{\prime} \\
\sum_{j:(j, i) \in L^{\prime}} q_{j i}-\sum_{j:(i, j) \in L^{\prime}} q_{i j}+Q g_{i}-D_{i}^{q}+s_{i}^{q}=0, \forall i \in N^{\prime} \\
V_{i}=V_{j}+\left(R_{i j} p_{i j}+X_{i j} q_{i j}\right) / V_{0}+\delta_{i j}, \forall(i, j) \in L^{\prime} \\
\left(-1+b_{i j}\right) V_{0} \leq \delta_{i j} \leq\left(1-b_{i j}\right) V_{0}, \forall(i, j) \in L^{\prime}, \forall t \\
V_{0} \sum_{k \in K_{b}} z_{i}^{k} \leq V_{i} \leq V_{0}, \forall i \in N^{\prime} \\
(1-\varepsilon) V_{0} \leq V_{i} \leq(1+\varepsilon) V_{0}, \forall i \in N^{\prime} \\
\sum_{k \in K} z_{i}^{k} \cdot P_{k \cdot \min } \leq P g_{i} \leq \sum_{k \in K} z_{i}^{k} \cdot P_{k \cdot \max }, \forall i \in N^{\prime} \\
\sum_{k \in K} z_{i}^{k} \cdot Q_{k \cdot \min } \leq Q g_{i} \leq \sum_{k \in K} z_{i}^{k} \cdot Q_{k \cdot \max }, \forall i \in N^{\prime} \\
0 \leq s_{i}^{p} \leq D_{i}^{p}, \forall i \in N^{\prime} \\
s_{i}^{q}=\frac{D_{i}^{q}}{D_{i}^{p}} s_{i}^{p}, \forall i \in N^{\prime} \\
-T_{i j}^{P} b_{i j} y_{i j} \leq p_{i j} \leq T_{i j}^{P} b_{i j} y_{i j}, \forall(i, j) \in L^{\prime} \\
-T_{i j}^{Q} b_{i j} y_{i j} \leq q_{i j} \leq T_{i j}^{Q} b_{i j} y_{i j}, \forall(i, j) \in L^{\prime}
\end{gathered}
$$

where $u:=\left(s^{p}, s^{q}, V_{i}, \delta_{i}, p_{i j}, q_{i j}, P g_{i}, Q g_{i}\right)$ denotes the postdisturbance decision variables. In this study, the linearized DistFlow model is adopted to formulate the power flow constraints in MGs. (13) and (14) calculate the active/reactive power balance flows for each node. (15) represents the node voltage relationship between two adjacent nodes, where $\delta_{i j}$ is a 
slack variable to make (15) valid when node $j$ does not belong to one MG but its parent node $i$ belongs to this MG. The constraint for $\delta_{i j}$ can be written as (16). The voltage level at each node is bounded by (17) and (18).

(19) and (20) describe the active/reactive power generation of DGs at each node. Additionally, (21) indicates that the active load shedding at node $n$ should be less than the active load demand. Analogously, we define the reactive load shedding at node $i s_{i}^{q}$ orthogonal to $s_{i}^{p}$ as defined by (22). Finally, (23) and (24) restrict the power flow on the lines.

The main difference between the proposed model and those in existing work is constraints (23)-(24). In the proposed model, the binary variable $y_{i j}$ is introduced, indicating whether the line between node $i$ and node $j$ is interrupted during the subsequent contingency. If line $(i, j)$ is damaged, $y_{i j}$ is zero, and constraints (23)-(24) enforce that $p_{i j}$ and $q_{i j}$ are equal to 0 , representing that no power can flow through this line.

\section{B. MILP Model for SSR}

After MGs are formed, the SSR is implemented in each MG. By generating sequential switching actions, the critical loads are picked up sequentially. The problem can be formulated as:

$$
\begin{aligned}
& \max \sum_{t \in T} \sum_{i \in N_{m}} w_{i} \cdot \Delta t \cdot P d_{i, t} \\
& x_{i i}^{R}=\left\{\begin{array}{l}
1, N_{i} \cap K_{b} \neq \varnothing \\
0, N_{i} \cap K_{b}=\varnothing
\end{array}\right. \\
& x_{i j}^{R}=0, i \neq j, N_{i} \cap K_{b}=\varnothing, N_{j} \cap K_{b} \neq \varnothing \\
& x_{i j}^{R}+x_{j i}^{R} \leq 1, i \neq j \\
& x_{i j}^{R} \leq L_{i j}, \forall(i, j) \in L^{\prime} \\
& \sum_{h}^{n^{m}} x_{h i}^{R . k} \leq 1, \forall i \\
& \sum_{j=1}^{n^{m}} x_{i j}^{R} \leq n^{m} \cdot \sum_{h=1}^{n^{m}} x_{h i}^{R}, \forall i \\
& T_{i i}^{R}-M\left(1-x_{i i}^{R}\right) \leq t_{i} \leq T_{i i}^{R}+M\left(1-x_{i i}^{R}\right), N_{i} \cap K_{b} \neq \varnothing \\
& T_{n}-M \sum_{h=1}^{n^{m}} x_{h i}^{R} \leq t_{i} \leq T_{n}+M \sum_{h=1}^{n^{m}} x_{h i}^{R} \\
& t_{h}+T_{h i}^{R}-M\left(1-x_{h i}^{R}\right) \leq t_{i} \leq t_{h}+T_{h i}^{R}+M\left(1-x_{h i}^{R}\right) \\
& \sum_{t=1}^{T_{n}} u_{i, t}^{L} \leq M \sum_{h=1}^{n_{c}} x_{h i}^{R} \\
& T_{n}-\sum_{t=1}^{T_{n}} u_{i . t}^{L} \geq t_{i} \\
& u_{i, t-1} \leq u_{i, t}, \forall t \geq 2 \\
& u_{i j . t}=u_{i . t} \cdot u_{j . t}, \forall(i, j) \in L^{\prime}, \forall t \\
& u_{i . t} \cdot P_{k \text { min }} \leq P g_{k . t} \leq u_{i . t} \cdot P_{k \text { max }}, N_{i} \cap N_{k} \neq \varnothing \\
& u_{i, t} \cdot Q_{k, \text { min }} \leq Q g_{k . t} \leq u_{i, t} \cdot Q_{k \text { max }}, N_{i} \cap N_{k} \neq \varnothing \\
& -R_{k}^{\text {ramp }} \leq P g_{k . t}-P g_{k . t-1} \leq R_{k}^{\text {ramp }}, N_{i} \cap N_{k} \neq \varnothing, \forall t \geq 2 \\
& P d_{i, t}=D d_{i}^{p}\left(\lambda_{i}^{U} u_{i, t}-\sum_{l=1}^{T} \Delta P d_{i}(l) \cdot u_{i, t-l+1}\right), \forall i \in N^{\prime}, \forall t \\
& Q d_{i . t}=D d_{i}^{q}\left(\lambda_{i}^{U} u_{i . t}-\sum_{l=1}^{T} \Delta Q d_{i}(l) \cdot u_{i, t-l+1}\right), \forall i \in N^{\prime}, \forall t
\end{aligned}
$$

$$
\begin{aligned}
& \sum_{j:(j, i) \in L^{\prime}} p_{j i . t}-\sum_{j:(i, j) \in L^{\prime}} p_{i j, t}+z_{i}^{k} \cdot P g_{k . t}-P d_{i, t}=0, \forall i \in N^{\prime}, \forall t \\
& \sum_{j:(j, i) \in L^{\prime}} q_{j i . t}-\sum_{j:(i, j) \in L^{\prime}} q_{i j, t}+z_{i}^{k} \cdot Q g_{k . t}-Q d_{i, t}=0, \forall i \in N^{\prime}, \forall t \\
& -T_{i j}^{P} u_{i j, t} \leq p_{i j, t} \leq T_{i j}^{P} u_{i j . t}, \forall(i, j) \in L^{\prime}, \forall t \\
& -T_{i j}^{Q} u_{i j . t} \leq q_{i j, t} \leq T_{i j}^{Q} u_{i j, t}, \forall(i, j) \in L^{\prime}, \forall t \\
& V_{0} \cdot z_{i}^{k} \leq V_{i, t} \leq V_{0}, \forall i \in n^{k}, k \in N_{b} \\
& V_{i . t}=V_{j . t}+\left(R_{i j} p_{i j . t}+X_{i j} q_{i j . t}\right) / V_{0}+\delta_{i j . t}, \forall i \in N^{\prime}, \forall t \\
& \left(-1+u_{i j . t}\right) V_{0} \leq \delta_{i j . t} \leq\left(1-u_{i j . t}\right) V_{0}, \forall(i, j) \in L^{\prime}, \forall t \\
& (1-\varepsilon) V_{0} \leq V_{i . t} \leq(1+\varepsilon) V_{0}, \forall i \in N^{\prime} \\
& \sum_{i \in N_{m}}\left(P d_{i, t}-P d_{i . t-1}\right) \leq \sqrt{2 M_{H}\left(f^{0}-f^{\mathrm{min}}\right) \cdot \sum_{g} u_{i, t} c_{g}}
\end{aligned}
$$

The objective function (25) is to optimize the restoration paths and energy management so that the maximum load can be restored during the SSR process. Considering the SR problem over a time span time $t=0$ to $t=T$, the time horizon can be denoted as $[0, T]$. In this paper, the time interval between two consecutive steps is assumed to be fixed.

(26)-(31) determine the restoration path. (26) indicates that a restoration path must start from a node with a black-start DG; (27) indicates that a restoration path cannot go through a black-start DG node to avoid forming a loop. (28) ensures that a node cannot be visited more than once, which also prevents a loop from occurring. (29) guarantees that the energization agent can only travel between the nodes that are connected by a line switch. (30) guarantees that a node can only be visited by one restoration path in an MG. Constraint (31) indicates that a downstream node can be restored only when its corresponding upstream node is restored, which guarantees that the structure of MGs is a radial network.

(32)-(34) describe the traveling time constraints. (32) means that if node $i$ contains a black start DG, it will be visited by an energization agent at time $T_{i i}^{R}$. (33) indicates that if node $i$ is not visited by any energization agent, $t_{i}$ will be set to $T^{R \cdot \max }$, which represents the maximum restoration time. Otherwise, $t_{i}$ will be calculated from (34), which forces $t_{i}=t_{h}+T_{h i}^{R}$ if the energization agent travels from node $h$ to node $i$.

(35)-(38) are the energization status constraints. (35) indicates that node $i$ can be energized only when it is visited by an energization agent. (36) denotes that the time when node $i$ is energized is constrained by the traveling time of an energization agent. (37) ensures that the node that has been restored will not be de-energized. (38) indicates that a line can be energized when both end nodes are energized.

(39)-(52) describes the MG operation constraints. (39) and (40) limit the active and reactive power outputs of a DG, and these constraints also force the output of a de-energized DG to 0 . (41) guarantees that the ramp rate of each DG is within the acceptable range. In this paper, we consider the loads under cold-load-pickup (CLPU) conditions. As introduced in [33], a typical delayed exponential CLPU curve is used to model the behavior of load demands at different time steps. (42) and (43) calculate the active and reactive power demands of load $i$ at step t. $D d_{i}^{p}$ and $D d_{i}^{q}$ are the restorable active and reactive power demands of load $i$, which are calculated in the MF stage (e.g. 
$\left.D d_{i}^{p}=D_{i}^{p}-s_{i}^{p}\right)$; The specific calculation process is illustrated in Appendix A. (44)(45) are the power flow equations. (46)(47) are the line capacity constraints. (48) sets the voltage of DGs at the root node as reference values. (49) calculates the voltage at other nodes. The slack variable $\delta_{i j}$ restricted in (50) takes care of the situation where nodes $i$ and $j$ do not belong to the same MG. (51) restricts the node voltage within an allowed deviation range. (52) restricts the amount of maximum allowable load pickup at a single time step to avoid large frequency deviations.

\section{Model Linearization}

Note that the above optimization model comprises some quadratic constraints, such as (7), (23), (24) and (38). Take constraint (23) as an example. Given that $b_{i j}$ and $y_{i j}$ are both variables, (23) is nonlinear. We define the auxiliary binary decision variable $b y_{i j}=b_{i j} y_{i j}$ to represent whether line $(i, j)$ is in the work state. The equation can be further converted to the following three linear inequalities:

$$
b y_{i j} \leq b_{i j}, b y_{i j} \leq y_{i j}, b y_{i j} \geq b_{i j}+y_{i j}-1
$$

In summary, (1)-(24) constitute the MF model and (25)-(52) constitute the SSR model. As a consequence of the linearization method mentioned above, the MF model is cast as a tri-level linear DR optimization model, and the SSR model is formulated as a MILP model.

\section{Solution Methodology}

\section{A. Ambiguity Set Construction in the MF Stage}

In the MF stage, a DR model is proposed, which considers the joint probability distributions of contingency based on the prediction information of line failure probability. An ambiguity set is constructed that matches the $N-k$ security criterion:

$$
D:=\left\{\mathbb{P} \in P(D(g)): 0 \leq E_{\mathbb{P}}[1-y] \leq \mu^{\max }\right\}
$$

where $P(D(g))$ represents the set of all probability distributions on a sigma-field of $D(g)$, which is the set of all subsets of $D(g)$. (54) implies that the marginal probability of each line $(m, n)$ not working during the restoration process has an upper limit $\mu^{\max }$. We designate $D(g)$ based on the $N-k$ contingency criterion [28]:

$$
D(g)=\left\{\sum_{(m . n) \in L^{\prime}}\left(1-y_{m n}\right) \leq k, y_{m n} \in\{0,1\}\right\}
$$

It is assumed by (55) that the number of fault lines is bounded by $k$, which can be calibrated based on reliability analyses of distribution lines during a contingency [31].

\section{B. Solution to the MF Stage Operation}

1) Problem Reformulation: We rewrite $\max _{\mathbb{P} \in D} E_{\mathbb{P}}[Q(g, y)]$ as:

$$
\begin{aligned}
\min _{\mathbb{P} \in D} & E_{\mathbb{P}}[Q(g, y)]=\min _{\mathbb{P}} \int_{D(g)} Q(g, y) d \mathbb{P} \\
\text { s.t. } & \int_{D(g)} d \mathbb{P}=1 \\
& \int_{D(g)}\left(1-y_{m n}\right) d \mathbb{P} \leq \mu_{m n}^{\max }, \forall(m, n) \in L^{\prime}
\end{aligned}
$$

According to [30], there exists a $\widehat{\mathbb{P}}$ that strictly satisfies (56.b) and (56.c). Thus, the Slater's condition holds between the problem (56) and the following dual formulation:

$$
\begin{aligned}
& \max _{\beta \geq 0, \lambda} \lambda-\sum_{(m, n) \in L^{\prime}} \mu_{m n}^{\max } \beta_{m n} \\
& \text { s.t. } \quad \lambda-\sum_{(m, n) \in L^{\prime}}\left(1-y_{m n}\right) \beta_{m n} \leq Q(g, y), \forall z \in D(g)
\end{aligned}
$$

where $\lambda$ and $\beta$ are dual variables associated with (56.b) and (56.c), respectively. In the dual formulation (57), the optimal $\lambda$ should satisfy

$$
\lambda=\min _{y \in D(g)}\left\{Q(g, y)+\sum_{(m, n) \in L^{\prime}}\left(1-y_{m n}\right) \beta_{m n}\right\}
$$

After the $\mathrm{g} \in \mathrm{G}$ is fixed, we have

$$
\begin{aligned}
& \min _{\mathbb{P} \in D} E_{\mathbb{P}}[Q(g, y)]= \\
& \max _{\beta \geq 0} \min _{y \in D(g)}\left\{Q(g, y)+\sum_{(m, n) \in L^{\prime}}\left(1-y_{m n}-\mu_{m n}^{\max }\right) \beta_{m n}\right\}
\end{aligned}
$$

After combining two maximizations, the original DR model is transformed into the classical RO problem as follows:

$$
\begin{array}{cc}
\max _{\beta \geq 0, g \in G} \min _{y \in D(g)} \max _{u \in H(g, y)} \sum_{n \in N^{\prime}} w_{n} \cdot\left(D_{n}^{p}-s_{n}^{p}\right)+ \\
\left.\sum_{(m, n) \in L^{\prime}}\left(1-y_{m n}-\mu_{m n}^{\max }\right) \beta_{m n}\right\} \\
\text { s.t. } \quad G=\{g \text { : Constraints (2)-(11) }\} \\
& H=\{u \text { : Constraints (13)-(24) }\}
\end{array}
$$

2) Model Decomposition: The RO model (60) is a tri-level model that can be solved through the C\&CG algorithm, which can decompose the original model into a master problem (MP) and a subproblem (SP).

The MP in the $l^{\text {th }}$ iteration is formulated as (61). In each iteration, a critical uncertain scenario is derived by solving the SP, and new constraints represented by $y^{l}$ are iteratively added to the MP:

$$
\begin{array}{ll}
\max _{\beta \geq 0, g \in G, \lambda, u^{k}} \theta+\sum_{(m, n) \in L^{\prime}}\left(1-\mu_{m n}^{\max }\right) \beta_{m n} \\
\text { s.t. } & \theta \leq \sum_{n \in N^{\prime}} w_{n} \cdot\left(D_{n}^{p}-s_{n}^{p . l}\right)-\sum_{(m, n) \in L^{\prime}} y_{m n}^{l} \cdot \beta_{m n} \\
& \forall y^{l} \in F, \quad \forall l=1, \ldots, r \\
& u^{l} \in H\left(g, y^{l}\right), \quad \forall l=1, \ldots, r
\end{array}
$$

where $F$ is the subset of all possible disturbance scenarios. The solution provides an upper bound for the original problem.

After the MP solution $g^{*}$ and $\beta^{*}$ are obtained, the following "min-max" bi-level model can be used to express the SP:

$$
\begin{aligned}
& \min _{y \in D\left(g^{*}\right)} \max _{u \in H\left(g^{*}, y\right)} \sum_{n \in N^{\prime}} w_{n} \cdot\left(D_{n}^{p}-s_{n}^{p}\right)-\sum_{(m, n) \in L^{\prime}} y_{m n} \cdot \beta_{m n}^{*} \\
& \text { s.t. (13) - (24) }
\end{aligned}
$$

The SP aims to derive a critical scenario of uncertainties. Since the inner problem is a linear programming problem, strong duality theory can be used to transform (62) into a single-level "min" problem. Note that the dual-objective function contains bilinear terms. We use the McCormick method introduced in [32] to further linearize such terms, and recast the $\mathrm{SP}$ as a mixed-integer linear program.

The solution of SP can be used to calculate the lower bound. The MP and SP are solved iteratively until the gap is reduced to an allowed termination threshold. After the MF model is solved, decision variables $\hat{g}$ are implemented to proactively form MGs.

\section{Solution to SSR Stage Operation}

For the SSR operation, restoration paths are generated, DG generation is dispatched and critical loads are picked up sequentially after the MGs are formed. In this paper, the SSR 
operation problem is formulated as a deterministic linear programming (LP) model as follows:

$$
\begin{array}{ll}
\max & c^{T} Y \\
\text { s.t. } & A X+B \hat{g} \leq p \\
& F X+G Y \leq v \\
& I X+J Y+H \hat{g}=w
\end{array}
$$

In (63), the decision variables include the restoration status, DG outputs, and load pickup statuses at each time step. Constraints (26)-(38) are grouped in (63.a); (39)-(43) and (46)-(52) are grouped in (63.b), where an equality constraint can be transformed into two inequality constraints; (63.c) is composed of (44)-(45). The formulation of (63) is an LP model that can be solved directly.

\section{Solution Procedure}

The complete solution procedure of the proposed algorithm for the two-step SR model can be described as follows.

$>$ Step 1: Initialization. Set the lower bound $\mathrm{LB}=-\infty$ and upper bound $\mathrm{UB}=+\infty$, set of contingencies $F=\varnothing$. Let the iteration index $l=1$. Pick a tolerance gap $\varepsilon$.

$>\quad$ Step 2: Solve the MP in (61) and obtain the optimal $g^{*}$, $\beta^{*}$ and the value of objMP. Update $\mathrm{UB}=\mathrm{objMP}$.

$>$ Step 3: Fix $g^{*}$ and $\beta^{*}$, and solve the "min-max"SP in (62) with the duality theory and McCormick method. Obtain optimal value of objSP and a contingency scenario $y^{l}$. Update $\mathrm{LB}=\max \left\{\mathrm{LB}, \mathrm{objSP}+\sum_{(m, n) \in L^{\prime}}\left(1-\mu_{m n}^{\max }\right) \beta_{\mathrm{mn}}^{*}\right\}$, Furthermore, update the contingency set $F=F \cup\left\{y^{k}\right\}$.

$>$ Step 4: If (UB-LB)/LB $\leq \varepsilon$, output the optimal $g$ and go to Step 6; Otherwise, update $l=l+1$, and go to the Step 5 .

$>$ Step 5: Create new variables $u^{l}$ and the corresponding constraints. Add them to the MP and go to Step 2.

$>\quad$ Step 6: With the optimized solution $\hat{g}$, solve the model in (63), and obtain the optimal SSR schemes.

\section{CAse Study}

\section{A. Simulation Setup}

The proposed MG-based SR simulation is conducted on the modified IEEE 37-node test system [12]. The distribution system is completely de-energized due to the first strike of the event, and the proposed SR strategy is implemented. Three permanent faults are applied to three locations (e.g. lines 701-799, 711-741 and 709-731), and then form the set $G \backslash G^{\prime}$.

TABLE I

DG PARAMETERS OF THE SYSTEM

\begin{tabular}{cccccc}
\hline \hline Parameter & DG1 & DG2 & DG3 & DG4 & DG5 \\
\hline Type & F(710) & F(704) & M & M & M \\
State & 1 & 1 & 1 & 0 & 0 \\
$P_{g}{ }^{\text {max }}(\mathrm{kW})$ & 70 & 80 & 90 & 60 & 50 \\
$P_{g^{r a m p}}(\mathrm{~kW} / \mathrm{min})$ & 25 & 20 & 10 & 10 & 10 \\
$Q_{g}{ }^{\text {max }}(\mathrm{kvar})$ & 60 & 75 & 80 & 50 & 45 \\
\hline \hline
\end{tabular}

The IEEE 37-node test system is modified to a balanced system, which includes two fixed DG units, and three MERs for allocation. The parameters of the DGs are shown in Table I, where the type "F" means the DG is pre-positioned and "M" indicates the DG is mobile. The 0/1 state reflects the black-start capacity of DGs, with " 1 " representing the DGs with black start capacity, and "O" indicating that the DG can only be energized after the cranking power is available. The active and reactive load demands at each node are randomly generated within intervals $[5,20] \mathrm{kW}$ and $[3,18] \mathrm{kVar}$. Similarly, weight coefficients, as well as CLPU load modeling parameters are generated using a uniform distribution as [33]. Parameters of the critical loads are shown in Table II. For simplicity, the energization time of each line is set to one time step. We also assume that the $\mu^{\max }$ of distribution lines in succeeding events follow independent Bernoulli distributions with different failure probabilities that vary randomly within interval $[0,0.03]$. For a practical system, determining the value of the failure probability is a complicated problem. Since the focus of this case study is to validate the SR model proposed, the method to calculate the line failure probability is out of the scope.

TABLE II

PARAMETERS OF CRITICAL LOADS

\begin{tabular}{ccccccc}
\hline \hline Node & Weight & $D^{P}(\mathrm{~kW})$ & $D^{Q}(\mathrm{kVar})$ & $\lambda^{U} / \lambda^{D}$ & $\tau$ (time step) & $\alpha$ \\
\hline N736 & 4.88 & 15 & 14 & $1.2 / 1.0$ & 1.5 & 0.69 \\
N711 & 4.52 & 15 & 13 & $1.3 / 1.0$ & 1.4 & 0.63 \\
N708 & 3.89 & 9 & 7 & $1.6 / 1.0$ & 1.6 & 0.69 \\
N709 & 3.61 & 11 & 9 & $1.6 / 1.0$ & 1.8 & 0.88 \\
N702 & 4.57 & 15 & 13 & $1.5 / 1.0$ & 1.6 & 0.92 \\
N707 & 5.16 & 8 & 5 & $1.5 / 1.0$ & 2.5 & 0.92 \\
\hline \hline
\end{tabular}

\section{B. Optimal Distribution System SR Scheme}

\section{1) MF Stage Results}

At this stage, (1)-(24) is solved to determine the MF scheme considering the potential contingency. In this simulation, we set the maximum number of fault lines to 2 for the system in succeeding events. To meet the power balance constraint, some noncritical loads are shaded in this stage. Table III shows the amount of active load shedding for each nodes.

TABLE III

AMOUNT OF ACTIVE LOAD SHEDDING

\begin{tabular}{cc|cc}
\hline \hline Node & $\begin{array}{c}\text { Amount of } \\
\text { Load Shedding(kW) }\end{array}$ & Node & $\begin{array}{c}\text { Amount of } \\
\text { Load Shedding(kW) }\end{array}$ \\
\hline N710 & 2.37 & N701 & 16 \\
N741 & 10 & N714 & 14 \\
N731 & 14 & N706 & 13 \\
N727 & 15 & N724 & 14 \\
N705 & 6 & Others & 0 \\
\hline \hline
\end{tabular}

The optimal MF scheme is shown in Fig. 4, in which the shaded areas represent the MG formed. As there are three DGs with black-start capacity, the system is proactively divided into three self-supplied MGs by opening the line switches. The radial topology is satisfied and each MG contains at least one DG. It can also be observed that the MERs tend to be located at nodes with critical loads. In this way, the contingencies that occur in the extended events are less likely to interrupt the power supply to restored critical loads.

In this case study, the gap $\varepsilon$ is set as $10^{-3}$. The value of UB and LB in this case study is shown in Fig. 5. It can be seen that the MP and SP provide upper and lower bounds of the original problem, respectively. The values of $\mathrm{UB}$ and $\mathrm{LB}$ are dynamically refined, thereby an optimal solution is generated. The proof for the guaranteed convergence of the proposed optimization algorithm is provided in Appendix B. 


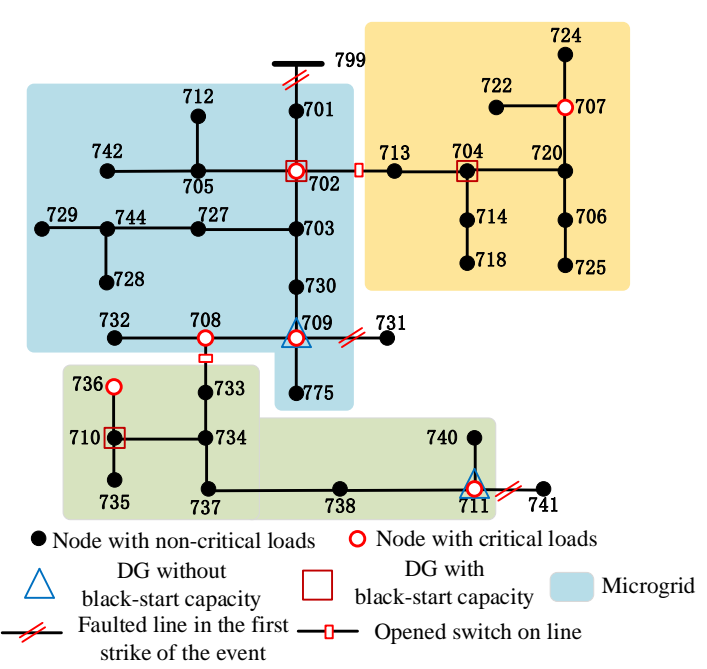

Fig. 4. Optimal MG topology for the37-bus distribution system

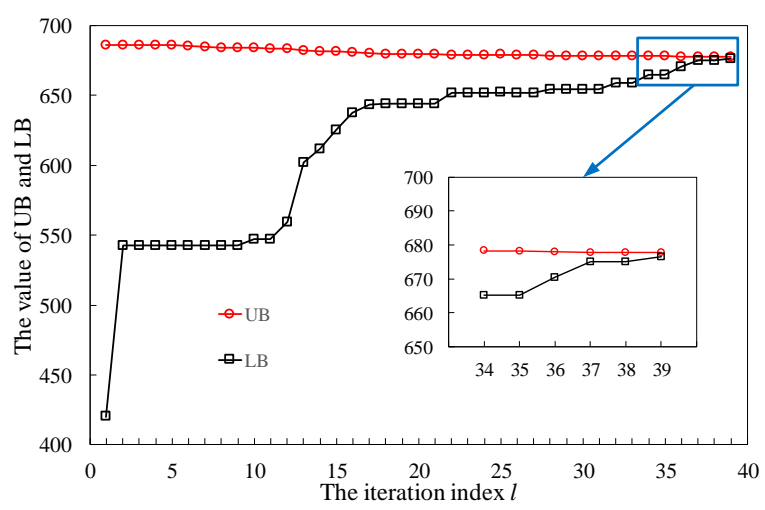

Fig. 5. The value of UB and LB in each iteration

Besides the optimal MF and load shedding schemes, it is also important to generate the worst-case contingency probability distribution. Some representative scenarios, as well as their corresponding probabilities, are presented in Table IV. Other scenarios with smaller probability values are omitted. The method to calculate the worst-case probability is presented in Appendix C. From this table, it can be observed that although Scenarios 1 has the largest contingency probability, load shedding due to the subsequent contingency in Scenario 1 will be much smaller than that in Scenario 6. This is because the interrupted lines in Scenarios 1 are at the end of the system. The representative worst-case contingency distribution can provide system operators a guideline on system vulnerability analyses.

TABLE IV

REPRESENTATIVE SCENARIOS AND THEIR CORRESPONDING PROBABILITIES

\begin{tabular}{ccc}
\hline \hline Scenario & Interrupted lines & Probability \\
\hline 1 & $709-775,744-729$ & 0.0147 \\
2 & $709-775,705-742$ & 0.0116 \\
3 & $710-735,734-737$ & 0.0035 \\
4 & $710-734,705-742$ & 0.0021 \\
5 & $733-708,708-709$ & 0.0009 \\
6 & $703-727,703-702$ & 0.0002 \\
\hline \hline
\end{tabular}

\section{2) SSR Stage Results}

After MGs are formed and the non-critical loads are shaded, the SSR process is performed to determine the switching sequences to restore the system step-by-step. The energization sequences are presented in Table V. As no more nodes are energized after the sixth step, only the first six steps are shown.
TABLE V

ENERGIZATION SEQUENCES IN MGS

\begin{tabular}{c|ccc}
\hline \hline \multirow{2}{*}{ Step } & MG1 & Node energized in & MG3 \\
\hline 1 & N710 & N702 & N704 \\
2 & N734,N735,N736 & N703,N705 & N713, N714,N720 \\
3 & N733,N737 & N730,N742,N712,N727 & N706,N718, N707 \\
4 & N738 & N744,N709 & N722, N724,N725 \\
5 & N711 & N728,N729,N798,N775 & \\
6 & N740 & N732 & \\
\hline \hline
\end{tabular}

The total amount of restored power in each MG for each step is shown in Fig. 6. Note that once a load is restored, the load demand will change according to the CLPU profile. It can also be observed from Table II and Fig. 6 that although some nodes are energized, the loads at these nodes cannot be restored immediately. For example, N735 and N736 are both energized at step 2, However, due to insufficient DG generation, L735 is restored at step 5. Moreover, the L734 cannot be restored until the DG5 is restored to avoid excessively frequency deviations.

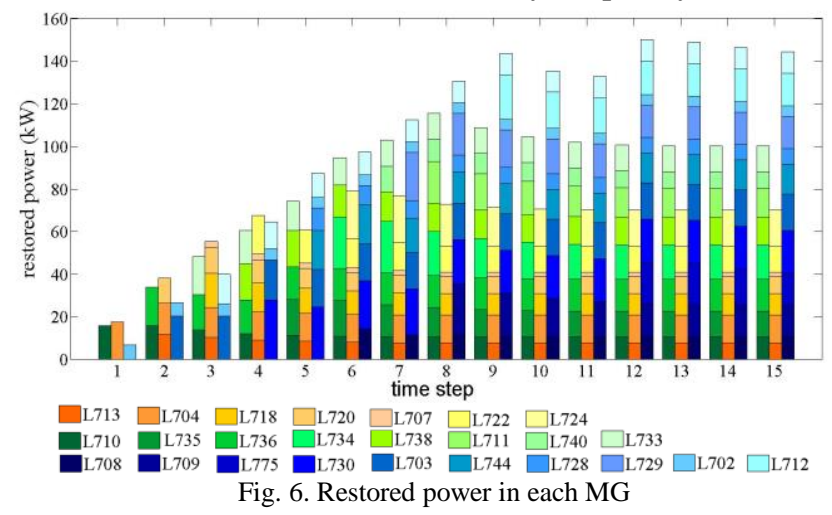

C. On the Value of MG formation and MERs

Three case studies were conducted to validate the value of optimally MG formation and MER deployments in systems. In Case 1, MERs are optimally allocated to form MGs by solving the proposed model. In Case 2, MEG units are randomly located and then the DR model is solved to form MGs. In Case 3, both the MEG locations and MG structures are randomly generated. The comparison of the expected weighted load restoration, and the number of iterations and the computational time in each case are listed in Table VI. To reflect the randomness, simulations are performed 10 times and the average values are listed.

TABLE VI

COMPARISONS OF THE VALUES IN THREE CASES

\begin{tabular}{cccc}
\hline \hline $\begin{array}{c}\text { Case } \\
\text { No. }\end{array}$ & $\begin{array}{c}\text { Expected load } \\
\text { restoration }\end{array}$ & $\begin{array}{c}\text { Number of } \\
\text { iterations }\end{array}$ & $\begin{array}{c}\text { Computational } \\
\text { time }\end{array}$ \\
\hline 1 & 677.73 & 39 & $133.53 \mathrm{~s}$ \\
2 & 618.21 & 28.8 & $92.74 \mathrm{~s}$ \\
3 & 526.57 & 16.2 & $56.7 \mathrm{~s}$ \\
\hline \hline
\end{tabular}

From Table VI, it can be concluded that: 1) the expected load restoration in Case 1 is more than that in Case 2, and it shows that optimally locating DERs can enhance the resilience of distribution systems. This is because faults of lines that connect DGs lead to a large amount of load shedding, while MERs can mitigate this influence by more flexibly constructing MGs; 2) Case 3 achieves the minimum value of expected load restoration. The result shows that, compared to networked MGs, the dynamic MG formation scheme can restore more critical loads. 


\section{The Effectiveness of the DR Approach}

To further demonstrate the effectiveness of the proposed MF method when facing upcoming disturbances, two comparative cases are conducted. In Case 4, the potential contingencies are ignored, and the MF scheme is obtained by solving a deterministic model. In Case 5, the RO model is conducted to form MGs. We first compare the expected load restorations in three cases. Then, we fix the MG configurations obtained by each model, and calculate the total weighted load restoration under a randomly simulated disturbance distribution within $D$. Table VII reports the expected load restoration (ELR), the load restoration under the worst-case scenario (LRW), and the out-of-sample average load restoration under randomly generated disturbances (ALR) in three cases by solving formulations (12)-(24). To reflect the randomness, we perform the simulations for 50 times.

TABLE VII

COMPARISONS OF LOAD RESTORATION IN THREE CASES

\begin{tabular}{cccc}
\hline \hline Case No. & ELR & LRW & ALR \\
\hline 1 & 677.73 & 516.18 & 579.24 \\
4 & 686.00 & 442.81 & 500.27 \\
5 & 584.47 & 480.63 & 528.55 \\
\hline \hline
\end{tabular}

According to Table VII, it can be found that: 1) although the MF scheme in Case 4 performs better prior to subsequent damage, it may trigger more shedding when faults actually occur in the succeeding extreme events. 2) Compared with the RO method, the proposed DR approach can restore more critical loads under the worst-case scenario and in the out-ofsample simulations. The reason is that probability distributions of line faults are considered, while the RO method merely focuses on the worst-case scenario. Therefore, the DR method can obtain a less conservative MF scheme and further enhance the resilience of MGs in succeeding extreme events.

\section{E. Sensitivity Analysis}

To investigate the impact of the DG capacity and the number of affected lines on the SR results, sensitivity analysis is performed. For comparison, the DG capacity is set from $70 \%$ to $100 \%$ of the DG capacity in Case 1. The number of affected lines allowed in succeeding events is set from 1 to 4 . The weighted load shedding under various scenarios is shown in Fig. 7.

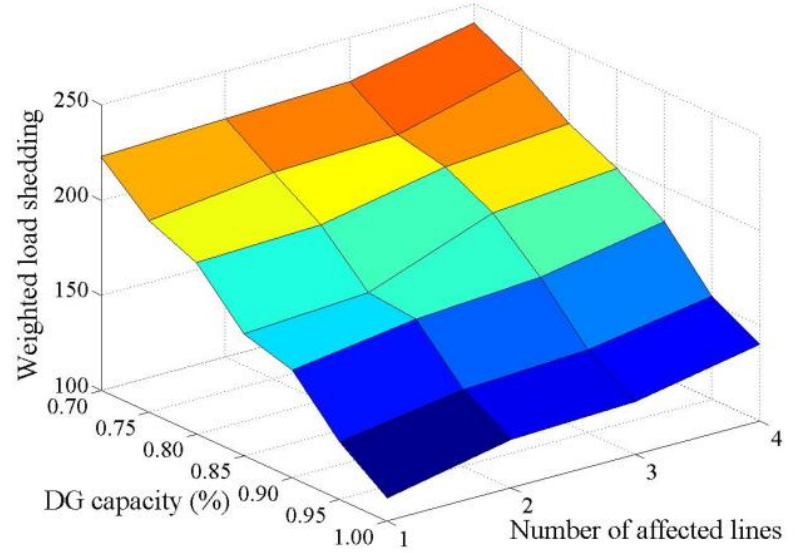

Fig. 7. The weighted load shedding under various DG capacity and number of affected lines
Obviously, the amount of expected weighted load shedding and the DG capacity are negatively correlated. This is because fewer loads can be supplied when the capacity of the DG decreases. In addition, the number of affected lines is also a critical parameter that affects the SR results. The weighted load shedding reduces as the number of faulty lines decreases. This is because more faulty lines will interrupt more restored loads in the succeeding events. Thus, the algorithm will make more conservative decisions to moderate this adverse effect.

\section{CONCLUSION}

This paper studies a two-stage SR approach that considers the random contingencies in succeeding events. The MF stage considers the uncertainty of line failure probability, and a DR approach is used to determine the MER location and the MG topology. The SSR stage optimizes the restoration paths, DG generation and load under CLPU conditions, aims at sequentially restoring the MG. Simulations are conducted on the IEEE 37-bus test system, and several conclusions can be obtained: 1) The optimal locations of MERs can improve the amount of weighted load restoration; 2) Compared with the traditional RO method, the proposed DR approach can provide less conservative MF scheme; 3 ) The expected weighted load shedding is sensitive to the DG capacity and the number of affected lines in succeeding events.

\section{APPENDIX A}

Take the active load demand under the CLPU condition as an example, a delayed exponential CLPU curve is shown in Fig. A1. Assume $N$ samples are collected. It can be seen from the CLPU curve that $\triangle P(k)$ represents the difference between two scale factors at $k_{t h}$ sample and $(k-1)_{t h}$ sample. $\Delta P(k)$ is calculated as (A1).

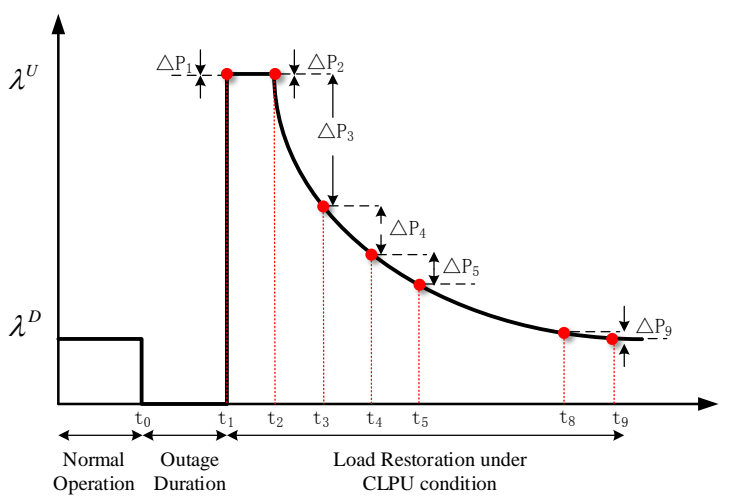

Fig. A1. The active load demand under CLPU condition

$$
\Delta \bar{P}(k)= \begin{cases}0 & k=1 \\ \bar{P}(k)-\bar{P}(k-1) & 1<k \leq T_{N}\end{cases}
$$

Based on the CLPU curve in Fig. A1, the scale factor of active load demand in $k_{\mathrm{th}}$ sample can be calculated as:

$$
\begin{gathered}
\bar{P}(k)=\left(\lambda^{D}+\left(\lambda^{U}-\lambda^{D}\right) e^{-\alpha \cdot \theta_{k}}\right) u\left(\theta_{k}\right) \\
+\lambda^{U}\left(1-u\left(\theta_{k}\right)\right), 1 \leq k \leq N \\
\theta_{k}=(k-1) \Delta t-\tau \\
u(x)= \begin{cases}1, & x>0 \\
0, & x \leq 0\end{cases}
\end{gathered}
$$


where $\lambda^{D}$ and $\lambda^{U}$ are diversified and undiversified loading factor for the load; $\alpha$ is the decay rate of the CLPU curve; $\theta(k)$ is the duration between the time step when the load begins gaining diversity and the $k_{t h}$ time step [33].

Based on (A1)-(A4), load demand under the CLPU condition can be formulated as constraints (42)-(43).

\section{APPENDIX B}

$D(g)$ in (55) is a finite set. The solution procedure converges when $\mathrm{LB}=\mathrm{UB}$, and the optimality of (60) is obtained.

Suppose at iteration $l, y^{l}$ can be obtained from (62), which leads to optimal $u$ and $\operatorname{ObjSP}^{l}$. It satisfies that:

$$
\begin{aligned}
\operatorname{ObjSP}^{l} & =\sum_{n \in N^{\prime}} w_{n} \cdot\left(D_{n}^{p}-s_{n}^{p . l}\right)-\sum_{(m, n) \in L^{\prime}} y_{m n}^{l} \beta_{m n}^{l} \\
& \geq \sum_{n \in N^{\prime}} w_{n} \cdot\left(D_{n}^{p}-\hat{s}_{n}^{p}\right)-\sum_{(m, n) \in L^{\prime}} y_{m n}^{l} \beta_{m n}^{l}
\end{aligned}
$$

Since $\mathrm{LB}=\max \left\{\mathrm{LB}, \mathrm{objSP}+\sum_{(m, n) \in L^{\prime}}\left(1-\mu_{m n}^{\max }\right) \beta_{\mathrm{mn}}^{*}\right\}$, it can be obtained that:

$$
L B^{l} \geq O \operatorname{ObjSP} P^{l}+\sum_{(m, n) \in L^{\prime}}\left(1-\mu_{m n}^{\max }\right) \beta_{m n}^{l}
$$

If $y^{l}$ has been identified in a previous iteration $l^{\prime}$ with $1 \leq l^{\prime} \leq l$, then it can be obtained that:

$$
\begin{aligned}
\theta^{l} & \leq \sum_{n \in N^{\prime}} w_{n} \cdot\left(D_{n}^{p}-s_{n}^{p}\right)-\sum_{(m, n) \in L^{\prime}} y_{m n}^{l^{\prime}} \cdot \beta_{m n} \\
& =\sum_{n \in N^{\prime}} w_{n} \cdot\left(D_{n}^{p}-s_{n}^{p}\right)-\sum_{(m, n) \in L^{\prime}} y_{m n}^{l} \cdot \beta_{m n} \\
& \leq \sum_{n \in N^{\prime}} w_{n} \cdot\left(D_{n}^{p}-s_{n}^{p . l}\right)-\sum_{(m, n) \in L^{\prime}} y_{m n}^{l} \cdot \beta_{m n}^{l}=O b j S P^{l}
\end{aligned}
$$

Hence, according to the model (61), it can be derived that:

$$
\begin{aligned}
U B^{l} & =o b j U^{l}=\theta^{l}+\sum_{(m, n) \in L^{\prime}}\left(1-\mu_{m n}^{\max }\right) \beta_{m n}^{l} \\
& \leq \operatorname{ObjSP}^{l}+\sum_{(m, n) \in L^{\prime}}\left(1-\mu_{m n}^{\max }\right) \beta_{m n}^{l}
\end{aligned}
$$

Then, based on the fact that $\mathrm{UB}^{l} \geq \mathrm{LB}^{l}$, and (B2) and (B4), we can obtain that $\mathrm{LB}^{l}=\mathrm{UB}^{l}$, and the convergence is proved.

\section{APPENDIX C}

Suppose that the C\&CG algorithm terminates at the Lth iteration with optimal $\hat{g}^{L}$ and $\left\{\hat{u}^{l}\right\}_{l=1, \ldots, L}$. The dual of Eq. (61) can be obtained as follows:

$$
\begin{aligned}
\min _{\eta \geq 0} & \sum_{l=1}^{L} \eta_{l} \cdot\left(\sum_{n \in N^{\prime}} w_{n} \cdot\left(D_{n}^{p}-s_{n}^{p . l}\right)\right) \\
\text { s.t. } & 1-\sum_{l=1}^{L} \eta_{l} \cdot y_{m n}^{l} \leq \mu_{m n}^{\max }, \forall(m, n) \in L^{\prime} \\
& \sum_{l=1}^{L} \eta_{l}=1
\end{aligned}
$$

where $\left\{\eta_{l}\right\}_{1=1, \ldots, L}$ represents the probability distribution of scenarios $\left\{y^{l}\right\}_{1=1, \ldots, L}$. According to the strong duality of linear programming, $(\mathrm{C} 1)$ is equivalent to the worst-case expectation formulation (60). Thus, $\left\{\eta_{l}\right\}_{l=1, \ldots, L}$ represents the worst-case contingency probability distribution.

\section{REFERENCES}

[1] R. Campbell, "Weather-related power outages and electric system resiliency," Congressional Research Service, Library Congr. Washington, DC, USA, 2012.

[2] M. A. Mohamed, T. Chen, W. Su, T. Jin, "Proactive resilience of power systems against natural disasters: A literature review, "IEEE Access, vol. 7, pp. 163778-163795, Nov. 2019.
[3] M. Shahidehpour, "Microgrids for enhancing the economics, reliability, and resilience of smart cities-An IIT experience," in Proc. Smart Grid Cond. (SGC), 2014, pp.1

[4] A. Gholami, T. Shekari, M. H Amirioun, et al., "Toward a consensus on the definition and taxonomy of power system resilience," IEEE Access, vol. 6, pp. 32035-32053, Jul. 2017.

[5] X. Wang, Z. Li, M. Shahidehpour, and C. Jiang, "Robust line hardening strategies for improving the resilience of distribution systems with variable renewable resources," IEEE Trans. Sustain. Energy, vol. 10, no.1, pp. 386395, Jan. 2019.

[6] M. H. Amirioun, F. Aminifar and H. Lesani, "Resilience-Oriented proactive management of microgrids against windstorms," IEEE Trans. Power Syst., vol. 32, no. 4, pp. 4275-4284, Jul. 2018.

[7] S. Lei, J. Wang, C. Chen, et al., "Mobile emergency generator prepositioning and real-time allocation for resilient response to natural disasters," IEEE Trans. Smart Grid, vol. 9, no. 3, pp. 2030-2041, May. 2018.

[8] S. Ma, S. Li, Z. Wang and F. Qiu, "Resilience-oriented design of distribution systems," IEEE Trans. Power Syt., vol. 34, no. 4, pp. 2880-2891, Jul. 2019.

[9] Y. Xu, C. C. Liu, K. P. Schneider, et al. "Microrids for service restoration to critical load in a resilient distribution system," IEEE Trans. Smart Grid, vol. 9, no. 1, pp. 416-437, Jan. 2018.

[10] F. Shen, J. C. López, Q. Wu, et al., "Distributed self-healing scheme for unbalanced electrical distribution systems based on alternating direction method of multipliers," IEEE Trans. Power Syst., vol. 35, no. 3, pp. 2190-2199, May. 2020.

[11] Z. Wang, B. Chen, J. Wang, C. Chen, "Network microgrids for self-healing power systems," IEEE Trans. Smart Grid, vol. 7, no. 1, pp. 310-319, Jan. 2016.

[12] C. Chen, J. Wang, F. Qiu and D. Zhao, "Resilient distribution system by microgrids formation after natural disasters," IEEE Trans. Smart Grid, vol. 7, no. 2, pp. 958-966, Mar. 2016.

[13] T. Ding, Y. L, Z. Bie and C. Chen, "A resilient microgrid strategy for load restoration considering master-slave distributed generators and topology reconfiguration,” Applied Energy, vol. 199, pp. 205-216, Aug. 2017.

[14] Z. Wang, J. Wang, "Self-healing resilient distribution systems based on sectionalization into microgrids," IEEE Trans. Power Syst., vol. 30, no. 6, pp. 3139-3149, Nov. 2015.

[15] S. Lei, J. Wang, C. Chen, et al., "Mobile emergency generator prepositioning and real-time allocation for resilient response to natural disasters," IEEE Trans. Smart Grid, vol. 9, no. 3, pp. 2030-2041, May. 2018.

[16] K. S. A. Sedzro, A. J. Lamadrid and L. F. Zuluaga, "Allocation of resource using a microgrid formation approach for resilient electric grids," IEEE Trans. Power syst., vol. 33, no. 3, pp. 2633-2643, May. 2018.

[17] Y. Wang, Y. Xu, J. He, et al., "Coordinating multiple sources for service restoration to enhance resilience of distribution systems," IEEE Trans. Smart Grid, vol. 10, no. 5, pp. 5781-5793, Sept. 2019.

[18] Z. Li, M. Shahidehpour, A. Alabdulwahab, and A. Abusorrah, "Analyzing locally coordinated cyber-physical attacks for undetectable line outages," IEEE Trans. Smart Grid, vol. 9, no. 1, pp. 35-47, Jan. 2018.

[19] L. Che, M. Shahidehpour, "Adaptive formation of microgrids with mobile emergency resources for critical service restoration in extreme conditions," IEEE Trans. Power syst., vol. 34, no. 1, pp. 742-753, Jan. 2019.

[20] J. Li, X. Y. Ma, C. C. Liu and K. P. Schneider, "Distribution system restoration with microgrids using spanning tree search," IEEE Trans. Power syst., vol. 29, no. 6, pp. 3021-3029, Nov. 2014.

[21] X. Chen, W. Wu, B. Zhang, "Robust restoration method for active distribution networks," IEEE Trans. Power syst., vol. 31, no. 5, pp. 40054015, Sept. 2016.

[22] A. Nasri, A. Abdollahi, M. P. Rashidinejad, "Probabilistic-proactive distribution network scheduling against a hurricane as a high impact-low probability event considering chaos theory," IET Gener., Transm. \& Disribt., 2020.

[23] B. Hu, L. Wu, "Robust SCUC considering continuous/discrete uncertainties and quick-start units: A two-stage robust optimization with mixed-integer recourse," IEEE Trans. Power syst., vol. 31, no. 2, pp. 1407-1419, Mar. 2016.

[24] D. N. Trakas, N. D. Hatziargyriou, "Optimal distribution system operation for enhancing resilience against wildfires," IEEE Trans. Power syst., vol. 33, no. 2, pp. 2260-2271, Mar. 2018. 
[25] K. S. A. Sedzro, X. Shi, A. J. Lamadrid, and L. F. Zuluaga, "A heuristic approach to the post-disturbance and stochastic pre-disturbance microgrid formation problem," IEEE Trans. Smart Grid, vol. 10, no. 5, pp. 5574-5586, Sept. 2019.

[26] A. Gholami, T. Shekari, S. Grijalva, "Proactive management of microgrids for resiliency enhancement: An adaptive robust approach, " IEEE Trans. Sustain. Energy, vol. 10, no. 1, pp. 470-480, Jan. 2019.

[27] G. Huang, J. Wang, C. Chen, et al., "Integration of preventive and emergency responses for power grid resilience enhancement," IEEE Trans. Power syst., vol. 32, no. 6, pp. 4451-4463, Nov. 2017.

[28] C. Zhao and R. Jiang, "Distributionally robust contingency-constrained unit commitment," IEEE Trans, Power Syst., vol. 33, no. 1, pp. 94-102, Jan. 2018.

[29] G. Zhang, F. Zhang, X. Zhang, Q. Wu and K. Meng, "A multi-disasterscenario distributionally robust planning model for enhancing the resilience of distribution systems," Int. Journal of Elec. Power \& Energy Syst., vol. 122, Nov. 2020.

[30] S. Babaei, R. Jiang and C. Zhao, "Distributionally robust distribution network configuration under random contingency," IEEE Trans, Power Syst., vol. 35, no. 5, pp. 3332-3341, Sept. 2020.

[31] Y. Sa, "Reliability analysis of electric distribution lines," Ph. D. dissertation, Canada: McGill University, 2002.

[32] G. P. McCormick, "Computability of global solutions to factorable nonconvex programs: Part I-convex underestimating problems," Math. Program., vol. 10, no. 1, pp. 147-175, 1976

[33] B. Chen, C. Chen, J. Wang, K. L. Butler-Purry, "Multi-time step service restoration for advanced distribution systems and microgrids," IEEE Trans. Smart Grid, vol. 9, no. 6, pp. 6793-6805, Nov. 2018. 